\title{
Sources of Resistance to Pathotype QCC of Puccinia graminis f. sp. tritici in Barley
}

\author{
Yue Jin, ${ }^{*}$ Brian J. Steffenson, and Thomas G. Fetch, Jr.
}

\begin{abstract}
The occurrence of a wheat stem rust (Puccinia graminis Pers.:Pers. f. sp. tritici Eriks. \& E. Henn.) pathotype (Pgt-QCC) with virulence for the Rpgl gene in barley (Hordeum vulgare L.) necessitated the search for resistant barley germplasm. From preliminary screenings of over 18000 barley accessions, 13 lines were identified as possessing resistance to pathotype QCC: 'Diamond', 'Hietpas 5', Q21861, PC 11, PC 84, PC 249, PC 250, CI 5541, PI 452406, PI 452421, PI 477843, PI 477854, and PI 477860. This study was conducted to further characterize the reaction of the selected lines to pathotype QCC. The reaction was assessed by evaluating infection types at the seedling stage and infection responses at the adult plant stage in the greenhouse, and by evaluating disease severity and infection responses at the adult plant stage in the field compared to susceptible cultivars. Most lines exhibited low to intermediate infection types at the seedlings stage and moderately resistant to moderately susceptible infection responses at the adult plant stage in the greenhouse experiments. Among the selected lines, Q21861 exhibited the highest level of resistance at both the seedling and adult plant stages. These lines may provide an adequate level of resistance to pathotype QCC for cultivar development.
\end{abstract}

$I^{\mathrm{N}}$ N CULTIVATED BARLEY, three genes are known to confer resistance to $P$. graminis f. sp. tritici, the wheat stem rust pathogen: Rpg1 derived from cultivars Chevron (CI 1111) (Shands, 1939), Peatland (CI 5267) (Powers and Hines, 1933), and Kindred (CI 6969) (Steffenson, 1992); Rpg2 from line 'Hietpas 5' (CI 7124) (Patterson et al., 1957); and Rpg3 from line PI 382313 (Jedel, 1990). Of the three described stem rust resistance genes, only Rpg 1 has been used to any extent in breeding programs. This gene confers resistance to a number of stem rust pathotypes (Immer et al., 1943) and has been incorporated into most commercial barley cultivars of the northern Great Plains (Steffenson, 1992). The widespread use of Rpg1 in breeding programs has resulted in a high level of genetic uniformity for stem rust resistance in barley. In 1989, the vulnerability of this gene deploy-

Dep. of Plant Pathology, North Dakota State Univ., Fargo, ND 58105 . Joumal Paper no. 2106 of North Dakota State Agric. Exp. Stn. Received 19 March 1993. *Corresponding author.

Published in Crop Sci. 34:285-288 (1994). ment scheme became apparent when a pathotype (PgtQCC) virulent for Rpg1 was detected throughout the northern Great Plains region (Martens et al., 1989; Roelfs et al., 1991). Pathotype QCC has become one of the most common virulence types in the stem rust population of the USA (Roelfs et al., 1993). Although severe and widespread epidemics of pathotype QCC have not occurred on barley, most cultivars remain vulnerable to stem rust because of their genetic uniformity for resistance.

To alleviate the threat of this rust pathotype in barley, sources of resistance must be identified for use in cultivar development. Over 18000 accessions of barley from the USDA National Small Grains Collection Research Facility (Aberdeen, ID) and cooperating organizations were evaluated for their reaction to pathotype QCC in greenhouse experiments at North Dakota State University (Fargo, ND) and in field nurseries at several locations. From these preliminary evaluations, 13 lines were selected for further study because they exhibited a lower level of rust infection compared to susceptible cultivars based on either greenhouse or field evaluations. This study was conducted to evaluate the reaction of these 13 lines to pathotype QCC at the seedling and adult plant stages in greenhouse and field experiments.

\section{MATERIALS AND METHODS \\ Plant Materials and Pathogen Isolate}

Pertinent information (pedigree, selection, or description and origin or source) on the 13 barley lines used in this study is given in Table 1. Cultivars Robust (PI 476976, possesses Rpg1) and Steptoe (CI 15229, does not possess any recognized stem rust resistance gene) were used as susceptible checks in these investigations. A culture of pathotype QCC of $P$. graminis f. sp. tritici, originally collected from cultivar Robust from Polk county (Minnesota) in 1990, was isolated and identified by Cereal Rust Laboratory (USDA-ARS, St. Paul, MN). Singleuredinial isolations were made by J.D. Miller (Northern Crop Science Laboratory, USDA-ARS, Fargo, ND) from this isolate to derive culture QCC-2. The culture QCC- 2 was confirmed to be pathotype Pgt-QCC based on its reaction on the 12 wheat differential lines of Roelfs and Martens (1988). The culture was increased on wheat 'Line E' (PI 357308) and was used throughout the greenhouse experiments. 
Table 1. Barley lines evaluated for resistance to pathotype QCC of Puccinia graminis f. sp. tritici.

\begin{tabular}{|c|c|c|}
\hline Line & Pedigree, selection or description & Origin (source) \\
\hline Diamond & Galt/Titan & $\begin{array}{l}\text { Canada (S. Kibite, Agricultural Canada Research Station, } \\
\text { Lacombe, Alberta, Canada) }\end{array}$ \\
\hline Q21861 & unknown & $\begin{array}{l}\text { CIMMYT (R. Dill-Macky and R. Rees, Queensland Wheat } \\
\text { Research Institute, Toowoomba, Australia) }\end{array}$ \\
\hline Hietpas 5 & selection from Oderbrucker & Wisconsin (USDA-ARS, NSGC, Aberdeen, ID) \\
\hline PC 11† & San Carlos//Gloria/Come“'S"/3/Cl2325//Boy*2/3*Surb & CIMMYT (S. Fox, Univ. Manitoba, Winnipeg, Canada) \\
\hline $\mathbf{P C 8 4 \dagger}$ & $\begin{array}{l}\text { Mola"S"/4/Brea“S"/DL70//Mozdosky/3/ } \\
\text { Nopal"S"/5/79W40762/6/Gloria“'S"/Copal“S" }\end{array}$ & CIMMYT (S. Fox, Univ. Manitoba, Winnipeg, Canada) \\
\hline PC 249† & $\begin{array}{l}\text { CI12823/CI585//CI9805.16D/3/Cita“S"/4/ } \\
\text { Gal//KL/2*CI 2376/3/Gloria "S" }\end{array}$ & CIMMYT (S. Fox, Univ. Manitoba, Winnipeg, Canada) \\
\hline PC $250 \dagger$ & ZARZA "S" & CIMMYT (S. Fox, Univ. Manitoba, Winnipeg, Canada) \\
\hline CI 5541 & Landrace accession & Ukraine (USDA-ARS, NSGC, Aberdeen, ID) \\
\hline PI 452406 & Unknown, breeding line & United Kingdom (USDA-ARS, NSGC, Aberdeen, ID) \\
\hline PI 452421 & Unknown, breeding line & United Kingdom (USDA-ARS, NSGC, Aberdeen, ID) \\
\hline PI 477843 & Landrace accession & Peru (USDA-ARS, NSGC, Aberdeen, ID) \\
\hline PI 477854 & Landrace accession & Peru (USDA-ARS, NSGC, Aberdeen, ID) \\
\hline PI 477860 & Landrace accession & Bolivia (USDA-ARS, NSGC, Aberdeen, ID) \\
\hline
\end{tabular}

†Resistant lines from the CIMMYT program were developed by Dr. Hugo E. Vivar (CIMMYT, Mexico).

\section{Field Evaluations}

Field nurseries were established at Fargo and Langdon, ND, in 1991 and 1992. Barley accessions were planted in hill plots (10-20 seeds per hill) in 1991 and in 1.8-m rows in 1992. Experiments were arranged in a randomized complete block design with four replications. Robust was used as a spreader row for pathotype QCC in the nurseries and was planted 10 to $14 \mathrm{~d}$ prior to the seeding date of the test entries. The nurseries were planted with a four-row cone planter, with test entries in the middle two rows and spreader plants in the outer rows. At each location, test entries were planted 15 to $20 \mathrm{~d}$ later than the recommended commercial planting date for barley to increase the chance for a natural stem rust epidemic. To augment the level of rust infection in the nurseries, test entries were inoculated with urediniospores collected from Robust spreader plants in the same nurseries when most of the test entries were between the late boot and early heading stages of development. Urediniospores were suspended in a light weight mineral oil (Soltrol 170, Phillips Petroleum, Borger, TX) at a concentration of $200 \mathrm{mg}$ urediniospores per $500 \mathrm{~mL}$ oil and inoculated with a Microfit sprayer (Micron Sprayers, Bromyard, UK) onto plants at a rate of approximately $1 \mathrm{~mL}$ per hill or $4 \mathrm{~mL}$ per short row. The stem rust on Robust spreader plants was identified to be pathotype QCC based on the reaction of the winter wheat cultivar McNair $701(\mathrm{Cl} 15288)$. This cultivar is resistant to pathotype QCC (exhibits low infection types) and susceptible to other common pathotypes in the Great Plains.

Infection response and disease severity (percentage of leaf sheath and stem area infected by rust) were assessed on each line between the soft and hard dough stages of development by the scales of Stubbs et al. (1986). The lowest, highest, and most common types of infection responses and estimated average rust severity were recorded on 20 to 30 randomly selected tillers in each hill or row plot. Tests for homogeneity of variance were nonsignificant between experiments; thus, rust severity data were pooled over locations and years for the analysis of variance and mean separation tests. Pathotypes of $P$. graminis f. sp. tritici present in each nursery were determined based on the seedling reaction of McNair 701. In the second year of the study, McNair 701 was planted in each nursery to monitor the presence of other pathotypes.

\section{Greenhouse Evaluations}

For seedling evaluations, four seeds of each line were sown in a plastic cone filled with a $3: 1$ peat moss:perlite mixture.
Each cone represented an experimental unit and treatments were replicated three times in a completely randomized design. Plants were grown in a greenhouse at $22 \pm 3{ }^{\circ} \mathrm{C}$ with 12 -h photoperiod. Seedling plants were inoculated with pathotype QCC when the primary leaves were fully expanded (about 1 wk after planting). These inoculations were made using a concentration of $6.0 \mathrm{mg}$ urediniospores per $\mathrm{mL}$ oil applied at an approximate rate of $2 \mu \mathrm{L}$ oil per plant. For adult plant evaluations, three to four seeds were sown in a clay pot $(15-\mathrm{cm}$ diam.) filled with the peat moss:perlite mixture and were grown at $22 \pm 3{ }^{\circ} \mathrm{C}$ in a greenhouse. Each pot represented an experimental unit and treatments were replicated four times in a randomized complete block design. Plants were inoculated when the main tiller was between the flowering to watery ripe stages of development. A rate of approximately $2 \mathrm{mg}$ urediniospores per $80 \mu \mathrm{L}$ oil was applied to the upper three internodes of each plant. After inoculation, plants (both seedling and adult) were incubated in an enclosed chamber where the humidity was maintained near saturation for $16 \mathrm{~h}$ at $21^{\circ} \mathrm{C}$ in the dark. Then, the plants were exposed to light $\left(160-230 \mu \mathrm{mol}\right.$ photon $\mathrm{m}^{-2}$ $\mathrm{s}^{-1}$ ) and allowed to dry for $4 \mathrm{~h}$ before being placed in a greenhouse.

Seedling plants were incubated in greenhouses at two temperature regimes $\left(23 \pm 5^{\circ} \mathrm{C}\right.$ and $\left.20 \pm 3{ }^{\circ} \mathrm{C}\right)$ with a 12 -h photoperiod supplied by sodium vapor bulbs $(430 \mathrm{~W}$ bulbs emitting 230-270 $\mu \mathrm{mol}$ photon $\mathrm{m}^{-2} \mathrm{~s}^{-1}$ ). Infection types were assessed on seedlings $12 \mathrm{~d}$ after inoculation using a rating scale based on the system of Stakman et al. (1962) as modified by Steffenson et al. (1993) for barley. Low infection types $(0,0$; 1,2 , or combinations thereof) were considered indicative of host resistance, whereas high infection types ( 3 and 4) were considered indicative of host susceptibility (Jin et al., 1993; Steffenson et al., 1993). Symbols + and - were used to denote more or less sporulation, respectively. Adult plants were incubated in greenhouses at $23 \pm 5^{\circ} \mathrm{C}$ under the same lighting conditions as described for the seedling tests. Infection responses (size and type of uredinia) were assessed on the leaf sheaths, stems, and peduncles of adult plants 18 to $20 \mathrm{~d}$ after inoculation by the scale of Stubbs et al. (1986).

\section{RESULTS}

The level of stem rust infection in the field varied among locations and years. Infection was low in the Langdon nursery (10-20\% on Robust checks), moderately high in the Fargo nursery in 1991 (40-70\% on 
Table 2. Adult plant infection response (IR), seedling infection type (IT), and terminal stem rust severity of barley lines to pathotype QCC of Puccinia graminis f. sp. tritici evaluated in the field and greenhouse in 1991 and 1992.

\begin{tabular}{|c|c|c|c|c|}
\hline \multirow[b]{2}{*}{ Line } & \multicolumn{2}{|c|}{ Field evaluations } & \multicolumn{2}{|c|}{ Greenhouse evaluations } \\
\hline & Adult IR $†$ & Disease severity(\%)‡ & Seedling IT§ & Adult IR \\
\hline Robust & $\mathrm{S}(\mathrm{MS} / \mathrm{S})$ & $40.0 \mathrm{a} \Upsilon$ & $2,1\left(1,2 / 2,3^{-}\right)$ & $\mathbf{S}(\mathbf{M S} / \mathbf{S})$ \\
\hline Steptoe & $\mathrm{S}(\mathrm{MS} / \mathrm{S})$ & $41.0 \mathrm{a}$ & $3,3^{+}\left(2,3 / 3^{+}\right)$ & S-MS (MS/S) \\
\hline Q21861 & MR (R/S) & $3.6 \mathrm{~b}$ & $0 ; 1\left(0 ; / 2,3^{-}\right)$ & R-MR (0/MS) \\
\hline Diamond & MR-MS (MR/S) & $5.9 \mathrm{~b}$ & $2,1\left(1,2 / 2,3^{-}\right)$ & MR-MS (R/S) \\
\hline Hietpas 5 & MS-S (MR/S) & $5.8 \mathrm{~b}$ & $2,3\left(2,3^{-} / 3^{-}, 2\right)$ & 0-MS (0/S) \\
\hline PC 11 & MR-MS (R/S) & $4.2 \mathrm{~b}$ & $1,2\left(1,2 / 3,3^{-}\right)$ & R-MR (R/MS) \\
\hline PC 84 & MR-MS (R/S) & $7.3 \mathrm{~b}$ & $2,3^{-}\left(1,2 / 2,3^{-}\right)$ & R-MR (R/S) \\
\hline PC 249 & MR-MS (R/S) & $3.6 \mathrm{~b}$ & $2,1\left(1,2 / 2,3^{-}\right)$ & MR-MS (MR/S) \\
\hline PC 250 & MR-MS (R/S) & $3.4 \mathrm{~b}$ & $2,1\left(1,2 / 2,3^{-}\right)$ & MR-MS (MR/S) \\
\hline CI 5541 & MS-S (MR/S) & $4.5 \mathrm{~b}$ & $1,2\left(1,2^{-} / 2,3^{-}\right)$ & MR (MR/MS) \\
\hline PI 452406 & MS-MR (R/S) & $7.4 \mathrm{~b}$ & $2,3^{-}\left(1,2 / 3^{-}, 2\right)$ & MS (MR/S) \\
\hline PI 452421 & MS (MR/S) & $9.0 \mathrm{~b}$ & $2,3^{-}\left(1,2 / 3^{-}, 2\right)$ & MR-MS (R/S) \\
\hline PI 477843 & MR-MS (R/S) & $5.9 \mathrm{~b}$ & $2,3^{-}\left(2,3^{-} / 3^{-}, 2\right)$ & MR-MS (MR/S) \\
\hline PI 477854 & MR-MS (R/S) & $6.9 \mathrm{~b}$ & $2,3^{-}\left(2,3^{-} / 3^{-}, 2\right)$ & MR-MS (MR/S) \\
\hline PI 477860 & MR-MS (R/S) & $6.9 \mathrm{~b}$ & $2,3-(2,3-/ 3-, 2)$ & MR-MS (MR/S) \\
\hline
\end{tabular}

† Infection responses (IRs) (size and type of uredinia) were used to characterize the reaction of barley lines to the stem rust pathogen at the adult plant stage where, $0=$ immune, $R=$ resistant, $M R=$ moderately resistant, $M S=$ moderately susceptible, and $S=$ susceptible following the rating scale of Stubbs et al. (1986). The one or two most common IRs observed on a line were listed first in order of prevalence followed by the lowest and highest IRs in parentheses.

¥ The mean disease severity was based on 16 observations, and each observation was obtained as an average of estimates from 20 to 30 tillers in a plot from the Fargo and Langdon nurseries in 1991 and 1992.

$\S$ Infection types (ITs) (size and type of uredinia) were used to characterize the reaction of barley lines to the stem rust pathogen at the seedling stage. Low ITs $(0,0 ;, 1$, and 2$)$ were considered indicative of resistance and high ITs $(3,4)$ of susceptibility following a system modified from Stakman et al. (1962). Symbols + and - denoted more or less sporulation of uredinia, respectively. The two most common ITs observed on a line were listed first in order of prevalence followed by the lowest and highest in parentheses.

T Means followed by the same letter were not significantly different at $\boldsymbol{P}=0.05$ using Duncan's new multiple range test.

Robust), and moderate in both nurseries in 1992 (20$40 \%$ on Robust). Pathotype determinations of 20 stem rust collections from Steptoe and Robust at each location in 1991 indicated that QCC was the only pathotype present in the nurseries. In 1992, no susceptible infection types were observed on McNair 701 in either of the two nurseries, indicating that QCC was the primary pathotype present.

The reactions of 15 barley lines (including checks) to pathotype QCC in the field are given in Table 2 (columns 2 and 3 ). Robust and Steptoe exhibited mostly susceptible infection responses, whereas most of the other lines exhibited moderately resistant to moderately susceptible infection responses. Moderately susceptible to susceptible infection responses were observed on Hietpas 5 and CI 5541. Q21861 exhibited the lowest infection response (moderately resistant), but the range was within that of most other lines. The range of infection responses overlapped among the lines because compatible uredinia occurred frequently on the peduncle and near the uppermost node on most lines, including Q21861. Mean disease severity on each of the 13 selected lines was significantly lower than on Robust and Steptoe. No significant difference for mean disease severity was detected among the 13 lines.

A wide range of infection types was observed on lines at the seedling stage (Table 2 columns 4 and 5). Intermediate infection types ( 2 to $3^{-}$) predominated on lines Hietpas 5, PC 84, PI 452406, PI 452421, PI 477843, PI 477854, and PI 477860, whereas low infection types (1 to 2) predominated on lines Diamond, PC 11, PC 249, PC 250, and CI 5541. The infection types on Q21861 were distinctly lower $(0$; to 1$)$ than on all other lines. Robust also exhibited low infection types at the seedling stage even though it is considered susceptible to pathotype QCC at the adult stage of development. Infection responses on adult plants in the greenhouse experiments generally corresponded to those observed in the field. A few lines, Q21861, PC 11, PC 84, and CI 5541, exhibited lower infection responses (resistant to moderately resistant) in the greenhouse than in the field.

\section{DISCUSSION}

Evaluations of more than 18000 accessions of cultivated barley and several wild barley species indicated that the frequency of resistance in Hordeum to pathotype QCC is very low. Of the 13 lines selected from preliminary germplasm evaluations, none possessed complete resistance to pathotype QCC (Table 2). A high degree of variation for disease reaction was observed in $H$. vulgare, but only a few lines exhibited levels of resistance that were considered sufficient for use in breeding programs. Q21861 exhibited the highest level of resistance to pathotype QCC among the 13 selected lines. This line also possesses resistance to several pathotypes of the wheat stem rust pathogen in Australia (Dill-Macky et al., 1992). Although Q21861 consistently exhibited a relatively high level of resistance in our experiments, severe stem rust infection was observed on this line in Brandon, Manitoba, in 1992 (W.G. Legge and D.E. Harder, 1992, personal communication). The reason for this discrepancy is unclear.

The ontogenetic expression of stem rust resistance can vary in different barley accessions. This was not thought to be a major factor in this study since most of the lines were similar in growth stage at the time of evaluation for terminal disease severity in the field. Environmental conditions, especially incubation temperature, can also affect the expression of resistance in barley to stem rust (Steffenson, 1992; Steffenson et al., 1993). Line Q21861 exhibited mostly susceptible infection types to pathotype 
QCC at the seedling stage when incubated at a temperature greater than $27^{\circ} \mathrm{C}$ (J.D. Miller, 1992, personal communication). Similar results were observed on all other selected lines in this study. The temperature sensitivity of resistance gene(s) in these lines may have contributed to the variable stem rust reactions observed in the field and greenhouse experiments.

Our preliminary data (Y. Jin and B.J. Steffenson, 1992, unpublished) indicate that resistance to pathotype QCC in Q21861, Diamond, Hietpas 5, PC 11, PC 84, and CI 5541 is simply inherited. Further studies are needed to genetically characterize these sources of resistance and their reaction to other pathotypes of $P$. graminis $\mathrm{f}$. $\mathrm{sp}$. tritici. If one or more genes for resistance to pathotype QCC is incorporated into cultivars already possessing $R p g 1$, possible losses in barley to stem rust should be averted.

\section{ACKNOWLEDGMENTS}

We thank Dr. H.E. Bockelman and Dr. D.M. Wesenerg (USDA-ARS, National Grains Collection and Research Facility, Aberdeen, ID) and other cooperating research groups and individuals for providing barley germplasm. We gratefully acknowledge the support of the American Malting Barley Association, North Dakota Barley Council, Minnesota Barley Research and Promotion Council, and North Dakota State University Agricultural Experiment Station.

\section{REFERENCES}

Dill-Macky, R., R.G. Rees, and W.J.R. Boyd. 1992. Sources of resistance to stem rust in barley. Plant Dis. 76:212.
Immer, F.R., J.J. Christensen, and W.Q. Loegering. 1943. Reaction of strains and varieties of barley to many physiologic races of stem rust. Phytopathology 33:253-254.

Jedel, P.E. 1990. A gene for resistance to Puccinia graminis $f$ sp. tritici in PI 382313. Barley Genet. Newsl. 20:43-44.

Jin, Y., B.J. Steffenson, and J.D. Franckowiak. 1993. Linkage between the Rpg1 gene for stem rust resistance and the $f 5$ locus on barley chromosome 1. Crop Sci. 33:642-643.

Martens, J.W., K.M. Dunsmore, and D.E. Harder. 1989. Incidence and virulence of Puccinia graminis in Canada on wheat and barley in 1988. Can. J. Plant Path. 11:424 430.

Patterson, F.L., R.G. Shands, and J.G. Dickson. 1957. Temperature and seasonal effects on seedling reactions of barley varieties to three races of Puccinia graminis f. sp. tritici. Phytopathology 47:395-402.

Powers, L., and L. Hines. 1933. Inheritance of reaction to stem rust and barbing of awns in barley crosses. J. Agric. Res. 46:1121-1129.

Roelfs, A.P., D.H. Casper, D.L. Long, and J.J. Roberts. 1991 Races of Puccinia graminis in the United States in 1989. Plant Dis. 75:1127-1130.

Roelfs, A.P., D.L. Long, and J.J. Roberts. 1993. Races of Puccinia graminis in the United States during 1990. Plant Dis. 77:125-128.

Roelfs, A.P., and J.W. Martens. 1988. An international system of nomenclature for Puccinia graminis f. sp. tritici. Phytopathology 78:526-533.

Shands, R.G. 1939. Chevron, a barley variety resistant to stem rust and other diseases. Phytopathology 29:209-211.

Stakman, E.C., D.M. Stewart, and W.Q. Loegering. 1962. Identification of physiologic races of Puccinia graminis var. tritici. USDA-ARS. E-617. U.S. Gov. Print. Office, Washington, DC.

Steffenson, B.J. 1992. Analysis of durable resistance to stem rust in barley. Euphytica 63:153-167.

Steffenson, B.J., J.D. Miller, and Y. Jin. 1993. Detection of the stem rust resistance gene Rpg1 in barley seedlings. Plant Dis. $76: 626-629$.

Stubbs, R.W., J.M. Prescott, E.E. Saari, and H.J. Dubin. 1986. Cereal Disease Methodology Manual. Centro Internacional de Mejoramiento de Maiz y Trigo (CIMMYT), Mexico. 